\title{
Video Article \\ Assembly of Gold Nanorods into Chiral Plasmonic Metamolecules Using DNA Origami Templates
}

\author{
Yike Huang ${ }^{1}$, Minh-Kha Nguyen ${ }^{1,2}$, Anton Kuzyk ${ }^{1}$ \\ ${ }^{1}$ Department of Neuroscience and Biomedical Engineering, Aalto University School of Science, Aalto FI-00076 \\ ${ }^{2}$ Faculty of Chemical Engineering, Ho Chi Minh City (HCMC) University of Technology, Vietnam National University - Ho Chi Minh City (VNU-HCM), Ho Chi Minh City \\ 700000
}

Correspondence to: Yike Huang at yike.huang@aalto.fi, Anton Kuzyk at anton.kuzyk@aalto.fi

URL: https://www.jove.com/video/59280

DOI: doi:10.3791/59280

Keywords: Chemistry, Issue 145, DNA nanotechnology, gold nanorods, DNA origami, self-assembly, chiral plasmonics, circular dichroism

Date Published: 3/5/2019

Citation: Huang, Y., Nguyen, M.K., Kuzyk, A. Assembly of Gold Nanorods into Chiral Plasmonic Metamolecules Using DNA Origami Templates. J. Vis. Exp. (145), e59280, doi:10.3791/59280 (2019).

\section{Abstract}

The inherent addressability of DNA origami structures makes them ideal templates for the arrangement of metal nanoparticles into complex plasmonic nanostructures. The high spatial precision of a DNA origami-templated assembly allows controlling the coupling between plasmonic resonances of individual particles and enables tailoring optical properties of the constructed nanostructures. Recently, chiral plasmonic systems attracted a lot of attention due to the strong correlation between the spatial configuration of plasmonic assemblies and their optical responses (e.g., circular dichroism [CD]). In this protocol, we describe the whole workflow for the generation of DNA origami-based chiral assemblies of gold nanorods (AuNRs). The protocol includes a detailed description of the design principles and experimental procedures for the fabrication of DNA origami templates, the synthesis of AuNRs, and the assembly of origami-AuNR structures. In addition, the characterization of structures using transmission electron microscopy (TEM) and CD spectroscopy is included. The described protocol is not limited to chiral configurations and can be adapted for the construction of various plasmonic architectures.

\section{Video Link}

The video component of this article can be found at https://www.jove.com/video/59280/

\section{Introduction}

DNA nanostructures, DNA origami in particular, have been widely used to arrange molecules and other nanoscale components (e.g., proteins and nanoparticles [NPs]), with nanometer precision into almost arbitrary geometries ${ }^{1,2,3,4,5}$. The ability to arrange metal NPs on DNA origami templates with a high yield and accuracy enables the fabrication of plasmonic structures with novel optical properties ${ }^{6,7,8,9,10}$ DNA origami technique is especially useful for the generation of chiral plasmonic structures, which require genuinely three-dimensional architectures ${ }^{11,12,13,14,15,16,17,18,19,20}$.

This protocol describes in detail the entire process of the fabrication of DNA origami-templated chiral assemblies of AuNRs. The software used for the design ${ }^{21}$ and structure prediction ${ }^{22,23}$ of DNA origami is intuitive and freely available. The origami fabrication and AuNR synthesis use common biochemistry lab equipment (e.g., thermocyclers, gel electrophoresis, hot plates, centrifuges). The structures are characterized using standard TEM and CD spectroscopy.

The fabrication of similar plasmonic nanostructures with top-down methods (e.g., electron beam lithography) would require rather complicated and expensive equipment. In addition, DNA origami templates provide the possibility to incorporate structural reconfigurability in plasmonic assemblies ${ }^{24,25,26,27,28,29,30,31,32,33}$, which is extremely challenging for structures fabricated with lithography techniques. Compared to other molecular-based approaches $34,35,36,37$, DNA origami-based fabrication provides a high level of spatial precision and programmability.

\section{Protocol}

\section{Design of the DNA origami}

1. Identify the desired relative spatial arrangement of AuNRs and the suitable shape of the DNA origami template (Figure 1A). Estimate the structural parameters of the AuNRs and the origami templates. Locate the approximate positions of staples that need further modification (Figure 1B).

2. Download and install caDNAno ${ }^{18}$ to design a DNA origami template. In caDNAno, route the scaffold and staple strands according to the desired shape of the template and generate the staple strands sequence by clicking Seq Tool. Click Paint Tool and mark the staple strands that require further modification (Figure 1C). 
3. Click Export Tool to export the DNA staple sequences (Figure 1C) to a .csv file.

4. Design double-stranded locks to fix the angle $\Theta$ between the two origami bundles. Depending on the relative orientation of the two bundles, the origami construct can adapt left- or right-handed $(\mathrm{LH} / \mathrm{RH})$ chiral spatial configuration (Figure 1B).

5. Import the staples' .csv file into a spreadsheet application. Add a polyA $A_{10}$ sequence at the end of the staples used for AuNR assembly (handles). Modify the staple strands on the designed lock sites with lock sequences.

NOTE: The assemblies in the representative results contain 36 handles protruding at the 3 ' end of the staple strands, 18 on each DNA origami bundle, equally distributed on two parallel helices every $21 \mathrm{nt}$. The distance between the first and the last handle position is $168 \mathrm{nt}$, approximately $57 \mathrm{~nm}$ (see the attached caDNAno file).

\section{Assembly of the DNA origami templates}

1. Prepare a working stock of staple strands (SM), including strands with handles and locks, by mixing equal amounts of concentrationnormalized staple oligonucleotides (e.g., $100 \mu \mathrm{M})$.

NOTE: Origami structures usually contain several hundreds of staple strands. Staples are typically purchased from vendors specializing in the chemical synthesis of DNA oligonucleotides in multiwell (e.g., 96-well) plates.

2. For $500 \mu \mathrm{L}$ of $10 \mathrm{nM}$ origami, mix $50 \mu \mathrm{L}$ of Tris-EDTA (TE, 10x), $100 \mu \mathrm{L}$ of $\mathrm{MgCl}_{2}(100 \mathrm{mM}), 25 \mu \mathrm{L}$ of $\mathrm{NaCl}(100 \mathrm{mM}), 175 \mu \mathrm{L}$ of $\mathrm{H}_{2} \mathrm{O}, 100 \mu \mathrm{L}$ of $\mathrm{SM}(0.5 \mu \mathrm{M}), 5 \mu \mathrm{L}$ of lock strands $(5 \mu \mathrm{M})$, and a $50 \mu \mathrm{L}$ scaffold $(100 \mathrm{nM})$.

3. Anneal the mixture in a thermocycler from $80^{\circ} \mathrm{C}$ to $20^{\circ} \mathrm{C}$ as described in Table 1.

\section{DNA origami purification}

NOTE: This section describes the protocol for agarose gel purification. DNA origami templates can also be purified using alternative approaches $^{38,39}$.

1. For $1 \%$ gel, dissolve $1 \mathrm{~g}$ of agarose in $100 \mathrm{~mL}$ of Tris-borate-EDTA (TBE, $0.5 \mathrm{x}$ ) by heating the mixture in a microwave oven. Add $10 \mu \mathrm{L}$ of $10,000 \times$ DNA stain according to the stain specification. To minimize the exposure to UV light at the extraction step (step 3.6), use a DNA stain that can be visualized under blue excitation.

2. Cool the solution to approximately $40^{\circ} \mathrm{C}$ and slowly add $1 \mathrm{~mL}$ of $\mathrm{MgCl}_{2}(1.3 \mathrm{M})$ while shaking. Cast gel and incubate for $30 \mathrm{~min}$ at room temperature.

3. Set the electrophoresis device and pour cold $\left(4^{\circ} \mathrm{C}\right)$ running buffer $(0.5 \mathrm{xTBE}$ with $11 \mathrm{mM} \mathrm{MgCl})$ into the gel box. Place the gel box in an ice water bath.

4. Add loading buffer to the origami samples (6x loading buffer contains $15 \%$ polysucrose 400 and $0.25 \%$ bromophenol blue in water). Load the samples into the wells with a proper volume according to the comb used (e.g., $50 \mu \mathrm{L}$ for an 8-well comb of $1.5 \mathrm{~mm}$ in thickness).

5. Run the electrophoresis for $2 \mathrm{~h}$ at $80 \mathrm{~V}$. NOTE: To characterize the origami and separate the open and closed structure, use $2 \%$ gel instead of $1 \%$ and prolong the running time to 4 h.

6. Image the gel with the gel imager (Figure 2). Use a blue light transilluminator to visualize the bands, cut the origami band, smash the gel on a parafilm, and extract the liquid. The recovery yield is approximately $40 \%$.

7. Pipette the liquid into a centrifugal filter unit and spin at $3,000 \times g$ for $5 \mathrm{~min}$. Measure the absorption of the origami solution at $260 \mathrm{~nm}$ with a UV-visible (UV-VIS) spectrometer. Estimate the concentration of origami using an extinction coefficient of $1.3 \times 10^{8} \mathrm{M}^{-1} \cdot \mathrm{cm}^{-1}$. NOTE: The typical concentration of origami solution after agarose gel purification is 1-2 nM.

8. Store the purified origami templates at $4{ }^{\circ} \mathrm{C}$ for later use.

\section{Synthesis of gold nanorods}

NOTE: The protocol for AuNR synthesis is adapted from previous literature ${ }^{40}$ with minor modifications.

1. Wash all glassware with aqua regia for $5 \mathrm{~min}$, rinse it with water, sonicate it with ultrapure water, and dry it before use.

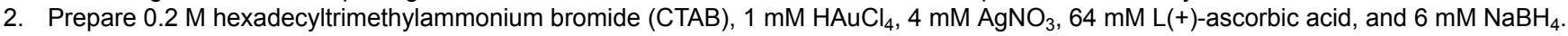
Use cold water $\left(4{ }^{\circ} \mathrm{C}\right)$ to dissolve $\mathrm{NaBH}_{4}$ and keep it in a fridge at $4{ }^{\circ} \mathrm{C}$. Ascorbic acid solution has to be freshly prepared.

CAUTION: CTAB is hazardous in case of skin contact (irritant), eye contact (irritant), ingestion, and inhalation. Wear suitable protective clothing. In case of insufficient ventilation, wear suitable respiratory equipment. $\mathrm{NaBH}_{4}$ is extremely hazardous in case of skin contact (irritant), eye contact (irritant), ingestion, and inhalation. Wear splash goggles, a lab coat, gloves, and a vapor and dust respirator. Be sure to use an approved/certified respirator or equivalent.

3. Prepare Au seeds.

1. Add $500 \mu \mathrm{L}$ of CTAB (0.2 M), $250 \mu \mathrm{L}$ of ultrapure water, and $250 \mu \mathrm{L}$ of $\mathrm{HAuCl}_{4}(1 \mathrm{mM})$ into a glass vial. Stir at $450 \mathrm{rpm}$ at room temperature for $5 \mathrm{~min}$.

2. Increase the stirring rate to $1,200 \mathrm{rpm}$. Add $100 \mu \mathrm{L}$ of cold $\mathrm{NaBH}_{4}$ solution $\left(6 \mathrm{mM}, 4^{\circ} \mathrm{C}\right)$. After $2 \mathrm{~min}$, stop the stirring and incubate the solution in a water bath at $30{ }^{\circ} \mathrm{C}$ for 30 min before use.

4. Prepare AuNRs.

1. Dissolve $0.55 \mathrm{~g}$ of $\mathrm{CTAB}$ and $0.037 \mathrm{~g}$ of 2,6-dihydroxybenzoic acid in $15 \mathrm{~mL}$ of warm water $\left(60-65^{\circ} \mathrm{C}\right)$ in a round-bottom flask. Cool down the solution to $30^{\circ} \mathrm{C}$, add $600 \mu \mathrm{L}$ of $\mathrm{AgNO}_{3}(4 \mathrm{mM})$, and stir at $450 \mathrm{rpm}$ for $2 \mathrm{~min}$. Then, leave the solution undisturbed for $15 \mathrm{~min}$ at $30^{\circ} \mathrm{C}$.

2. Add $15 \mathrm{~mL}$ of $\mathrm{HAuCl}_{4}(1 \mathrm{mM})$ to the solution, and stir at $450 \mathrm{rpm}$ for $15 \mathrm{~min}$. Add $120 \mu \mathrm{L}$ of $\mathrm{L}(+)$-ascorbic acid (64 mM), and then, immediately, stir at $1,200 \mathrm{rpm}$ for $30 \mathrm{~s}$. Add $12 \mu \mathrm{L}$ of Au seeds, and keep stirring at 1,200 rpm for $30 \mathrm{~s}$.

3. Incubate the solution in a water bath at $30{ }^{\circ} \mathrm{C}$ for $18 \mathrm{~h}$. Do not disturb the solution and use a cap to close the flask. 
4. Transfer the resultant solution to centrifuge tubes, and centrifuge at $9,500 \times g$ for $12 \mathrm{~min}$ at $20^{\circ} \mathrm{C}$. Discard the supernatant, disperse the pellet in $20 \mathrm{~mL}$ of ultrapure water, and perform one more centrifugation step.

5. Disperse the final pellet in $3 \mathrm{~mL}$ of distilled water. Estimate the concentration of AuNRs from a UV-VIS absorption measurement using the extinction coefficient for the longitudinal plasmon resonance. The extinction coefficient can be predicted using AuNR shape parameters ${ }^{41}$. Store the AuNRs at $4{ }^{\circ} \mathrm{C}$ for further use.

\section{Functionalization of gold nanorods with single-stranded DNA}

NOTE: This section describes the protocol for AuNR functionalization with single-stranded DNA (ssDNA), following the so-called low pH route adapted from previous literature ${ }^{42}$. The AuNRs covered with DNA are purified by centrifugation; alternatively, the purification can be performed using agarose gel electrophoresis.

1. Incubate $20 \mu \mathrm{L}$ of thiol-functionalized polyT DNA strands $(1 \mathrm{mM})$ with $20 \mu \mathrm{L}$ of freshly prepared tris(2-carboxyethyl)phosphine hydrochloride (TCEP, $14 \mathrm{mM}$ ) for $1 \mathrm{~h}$ to reduce disulfide bonds.

NOTE: The thiol groups form bonds with AuNRs, and the polyT sequence hybridizes with the poly $\mathrm{A}_{10}$ handle on the origami, in which too many or too few base pairs may lead to a malfunction or an unstable assembly.

CAUTION: TCEP can cause severe skin burns and eye damage. Wear protective gloves/protective clothing/eye protection/face protection

2. Mix $150 \mu \mathrm{L}$ of AuNRs $(10 \mathrm{nM})$ and $40 \mu \mathrm{L}$ of TCEP-treated thiol-DNA (0.5 mM). Add $1 \%$ sodium dodecyl sulfate (SDS) to the AuNR solution to reach a final SDS concentration of $0.05 \%$. Adjust the $\mathrm{pH}$ to $2.5-3$ with $\sim 1 \mu \mathrm{L}$ of $\mathrm{HCl}(1 \mathrm{M})$.

3. Incubate for $2 \mathrm{~h}$ while shaking at $70 \mathrm{rpm}$.

NOTE: The AuNR-to-DNA ratio should be in the order of 1:5,000-15,000, depending on the size of the rods. For the AuNRs (70 $\mathrm{nm} \times 30 \mathrm{~nm}$ ) prepared following the protocol described in section 4, a 13,000 excess of thiol-DNA is recommended.

4. Add $\mathrm{NaCl}$ to reach a final $\mathrm{NaCl}$ concertation of $0.5 \mathrm{M}$ and incubate for $4 \mathrm{~h}$ at room temperature while shaking at $70 \mathrm{rpm}$. NOTE: A color change at this step may indicate a failed DNA functionalization.

5. Adjust the $\mathrm{pH}$ to $\sim 8.5$ with TBE buffer (10x) and incubate overnight.

6. Wash the DNA-AuNRs $4 x$ by mixing the samples with $1 \mathrm{~mL}$ of washing buffer $(0.5 x$ TBE with $0.1 \%$ SDS), and centrifuge at $7,000 \times g$ for 30 min. Remove the supernatant and resuspend the DNA-AuNRs in the remaining liquid $(\sim 40 \mu \mathrm{L})$. Estimate the concentration of DNA-AuNRs from a UV-VIS absorption measurement as described in step 4.4.5.

NOTE: The solution might become slightly 'cloudy' at steps 5.3-5.4 due to the CTAB replacement from the surface of the AuNRs by thiolDNA. The solution should become clear upon warming up to $\sim 35^{\circ} \mathrm{C}$ for $5 \mathrm{~min}$.

\section{Assembly of gold nanorods on DNA origami templates}

1. Add $\mathrm{MgCl}_{2}$ to the solution of purified DNA-AuNRs, to a final concentration of $10 \mathrm{mM}$. Mix the purified DNA-AuNRs and origami to a $10: 1$ ratio. NOTE: A lower ratio may decrease the product yield ${ }^{43}$.

2. Anneal the mixture in a mixer with a temperature control from $40{ }^{\circ} \mathrm{C}$ to $20^{\circ} \mathrm{C}$ while shaking at $400 \mathrm{rpm}$, following the procedure in Table 2 . NOTE: For CD characterization, the sample can be measured after this step without further purification.

3. Use $0.7 \%$ agarose gel electrophoresis $(3.5 \mathrm{~h}$ at $80 \mathrm{~V})$ to purify the final origami-AuNR structures.

4. Use a white light transilluminator for imaging. Cut the product band (origami-AuNR dimer) (Figure 3), smash the gel on a parafilm, and extract the liquid. Pipette the liquid into a centrifugal filter unit and spin at 3,000 $\mathrm{g} g$ for $5 \mathrm{~min}$. Resuspend the origami-AuNRs in the solution. The recovery yield from the gel is approximately $50 \%$.

5. Estimate the concentration of the origami-AuNR structures from a UV-VIS absorption measurement as described in step 4.4.5.

\section{Transmission electron microscopy imaging}

NOTE: This uranyl formate (UFo) staining protocol is adapted from previous literature ${ }^{44}$.

1. Mix $200 \mu \mathrm{L}$ of UFo solution $(0.75 \%)$ and $1 \mu \mathrm{L}$ of $\mathrm{NaOH}(5 \mathrm{M})$ and vortex immediately for $2-3$ min. Centrifuge the stain solution for $3-4$ min at $14,000 \times g$. Protect the stain from light exposure (e.g., by wrapping it in aluminum foil).

CAUTION: UFo is toxic if inhaled or swallowed and can cause eye irritation. In the case of brief exposure or low pollution, use a respiratory filter device. In the case of intensive or longer exposure, use a self-contained respiratory protective device. Wear gloves. The glove material has to be impermeable and resistant to UFo and its solutions. Wear tightly sealed goggles.

2. Glow-discharge carbon/formvar-coated TEM grids for $6 \mathrm{~s}$ just before use to increase hydrophilicity and promote the sticking of the structures. Pipette $5 \mu \mathrm{L}$ sample drops on the TEM grid, incubate for 5-8 min, and remove the drop by gently touching a filter paper with the edge of the grid.

3. Pipette one big $(\sim 20 \mu \mathrm{L})$ and one small $(\sim 10 \mu \mathrm{L})$ drop of the stain solution on a parafilm.Put the grid on the small stain solution drop and dry immediately by touching the filter paper with the edge of the grid. Then, put it on the big stain solution drop for $30 \mathrm{~s}$.

4. Remove the liquid on the grid by touching the filter paper with the edge of the grid. Place the grid in the grid holder. Wait for the grid to dry for at least $10 \mathrm{~min}$.

5. Characterize the samples of origami (Figure 4), AuNRs (Figure 5), and origami-AuNRs (Figure 6) by TEM.

\section{Circular dichroism measurement}

1. Purge the CD spectrometer with $\mathrm{N}_{2}$ for 20 min.

NOTE: Most of the CD spectrometers require purging with $\mathrm{N}_{2}$ before lamp ignition. Check the CD spectrometer manual.

2. Set the bandwidth, scanning range, and acquisition step.

NOTE: The scanning range depends on the optical properties of AuNRs, which depend on the size of the AuNRs. 
3. Measure blank CD with buffer.

4. Measure the CD spectra of origami-AuNR samples (Figure 7).

NOTE: Use quarts or glass cuvettes for CD measurement. Plastic cuvettes are unsuitable for CD spectroscopy. Also, most CD spectrometers allow the simultaneous acquisition of absorption and CD data.

\section{Representative Results}

TEM images of DNA origami templates, AuNRs, and final origami-AuNR assemblies are shown in Figure 4, Figure 5, and Figure 6A,

respectively. Due to their binding preference to TEM grids, origami-AuNR assemblies are usually seen as parallel origami bundles and rods

(Figure 6A). Thermal annealing is required for the correct alignment of AuNRs on origami templates (Figure 6A,B). The protocol enables high yields of the assembly of AuNRs into chiral metamolecules with strong plasmonic CD responses (Figure 7).

\begin{tabular}{|l|l|}
\hline Temperature $\left({ }^{\circ} \mathrm{C}\right)$ & Time \\
\hline 80 & $15 \mathrm{~min}$ \\
\hline $79-71$ & $1{ }^{\circ} \mathrm{C} / 1 \mathrm{~min}$ \\
\hline $70-66$ & $1{ }^{\circ} \mathrm{C} / 5 \mathrm{~min}$ \\
\hline $65-60$ & $1{ }^{\circ} \mathrm{C} / 30 \mathrm{~min}$ \\
\hline $59-37$ & $1{ }^{\circ} \mathrm{C} / 60 \mathrm{~min}$ \\
\hline $36-30$ & $1^{\circ} \mathrm{C} / 15 \mathrm{~min}$ \\
\hline $29-20$ & $1^{\circ} \mathrm{C} / 5 \mathrm{~min}$ \\
\hline 20 & Hold \\
\hline
\end{tabular}

Table 1: Temperatures and rates for the thermal annealing of DNA origami templates.

\begin{tabular}{|l|l|}
\hline Temperature $\left({ }^{\circ} \mathbf{C}\right)$ & Time $(\mathbf{m i n})$ \\
\hline 40 & 130 \\
\hline 36 & 180 \\
\hline 32 & 180 \\
\hline 22 & Hold \\
\hline
\end{tabular}

Table 2: Temperatures and holding times for the annealing of AuNRs and DNA origami templates. The cooling rate between the steps is set at $0.1^{\circ} \mathrm{C} / \mathrm{min}$. The DNA origami-AuNR samples are annealed while shaking at $400 \mathrm{rpm}$.

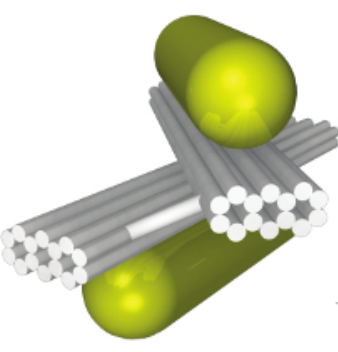

B
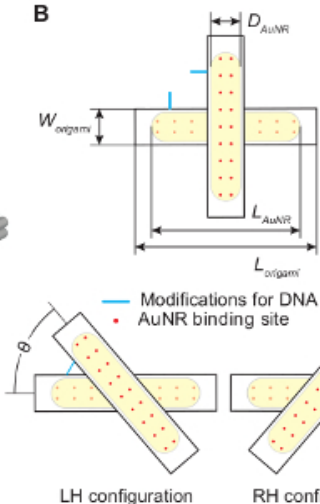
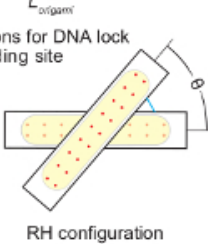

ORIGAMI DESING (CADNANO)

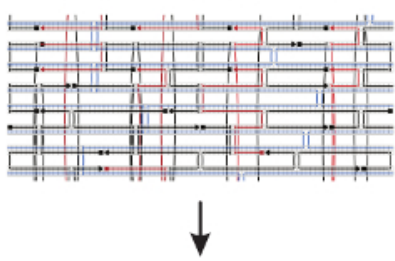

DNA SEQUENCES

ATACGTATCGGGATCT CTATTCGGCATATTCG AGTCGATCGATCGACT
TACGTAGCTACGATCE TACGTAGCTACGATCG ACTACCATCACTCGATC GCTGTAATTGCTTAGC

Figure 1: Design of DNA origami-templated chiral metamolecules. (A) Identify the desired relative spatial arrangement of gold nanorods (AuNRs) and a suitable shape of the DNA origami template. (B) Estimate the structural parameters of the AuNRs $\left(D_{\text {AuNR, }}, L_{\text {AuNR }}\right)$ and the origami template $\left(W_{\text {origami }}, L_{\text {origami }}, \Theta\right)$. Locate the approximate positions of the staples that need further modification. (C) Design of DNA origami templates using caDNAno. Please click here to view a larger version of this figure. 
A

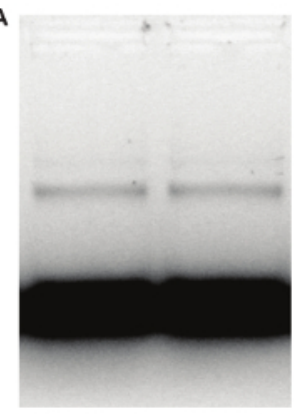

loading well

origami

excess staple strands
B

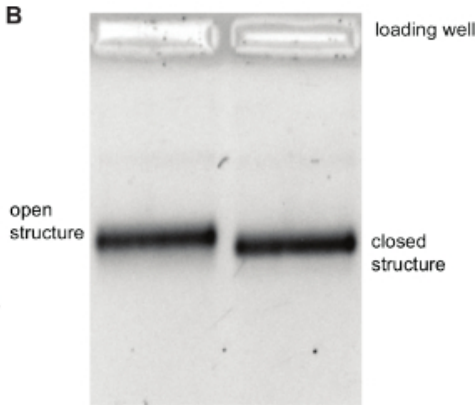

Figure 2: The agarose gel electrophoresis of origami. (A) Purification with $1 \%$ agarose gel electrophoresis for $2 \mathrm{~h}$ at $80 \mathrm{~V}$. (B) Characterization with $2 \%$ agarose gel electrophoresis for $4 \mathrm{~h}$ at $80 \mathrm{~V}$. Please click here to view a larger version of this figure.

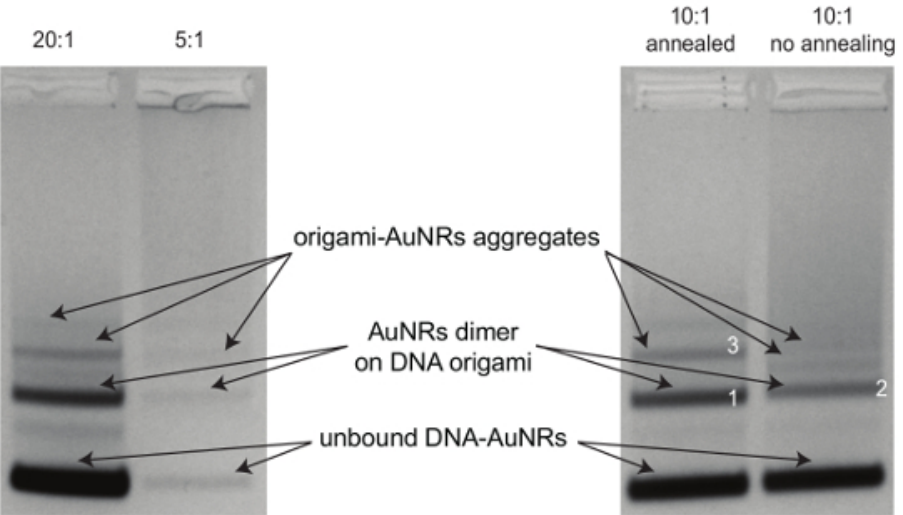

Figure 3: The agarose gel electrophoresis purification of origami-AuNRs. Gel $(0.7 \%)$ was run for $3.5 \mathrm{~h}$ at $80 \mathrm{~V}$ for the samples prepared following the assembly procedure with different DNA-AuNR-to-origami ratios (20:1, 5:1) and samples (10:1 DNA-AuNRs-to-origami ratio) with/ without annealing procedure. For TEM images of the samples in bands 1, 2, and 3, see Figure 6 . Please click here to view a larger version of this figure.

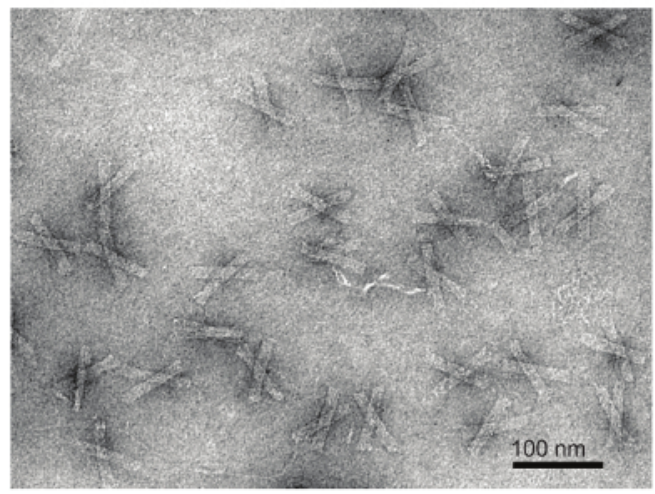

Figure 4: Representative TEM image of the DNA origami templates. The origami structure consists of two 14-helix bundles (80 $\mathrm{nm} \times 16 \mathrm{~nm} \mathrm{x}$ $8 \mathrm{~nm}$ ) linked together by the scaffold strand. Please click here to view a larger version of this figure. 


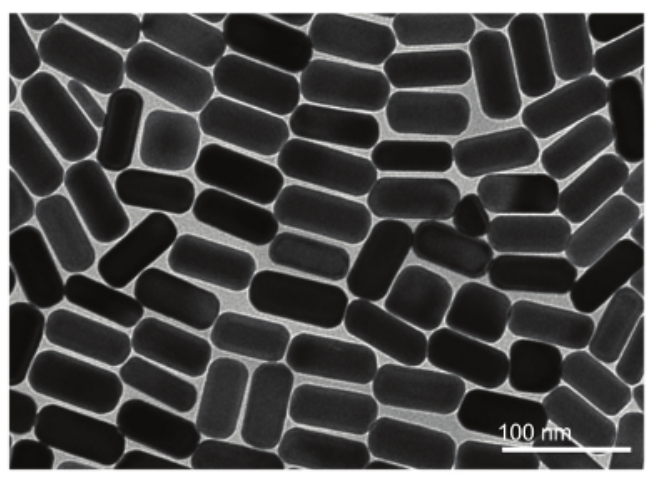

Figure 5: Representative TEM image of the AuNRs. The average dimensions of synthesized AuNRs are $70 \times 30 \mathrm{~nm}$. Please click here to view a larger version of this figure.

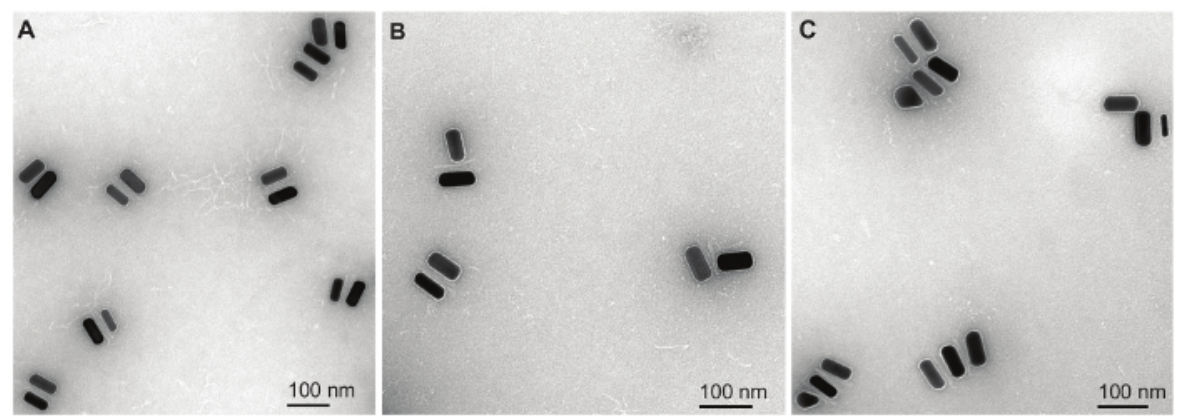

Figure 6: TEM images of origami-AuNR assemblies. (A) AuNR dimers on origami after annealing (band 1 in Figure 3). (B) AuNR dimers on origami without annealing (band 2 in Figure 3). (C) Origami-AuNR aggregates (band 3 in Figure 3). Please click here to view a larger version of this figure.

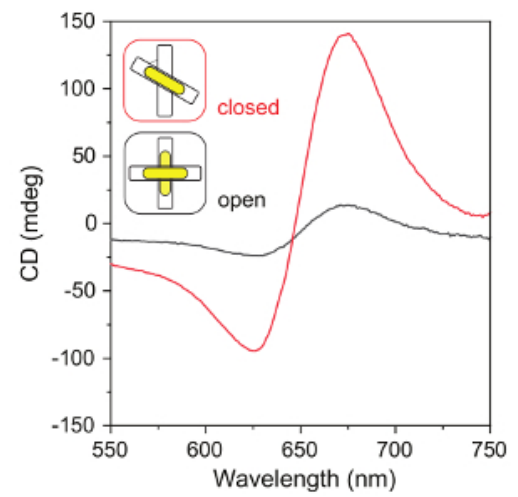

Figure 7: CD spectra of the origami-AuNR assemblies. The CD spectra of the closed structures (the origami templates fixed by lock strands into a right-handed configuration, with $50^{\circ}$ between two origami bundles) and the open structure (the origami templates without lock strands). Please click here to view a larger version of this figure.

\section{Discussion}

The protocol introduces the whole workflow of design, assembly, purification, and characterization of DNA origami-based chiral assemblies of AuNRs. The DNA origami templates used in the protocol are particularly suitable for the fabrication of stimuli-responsive assemblies. Various types of responses and functionalizes can be incorporated into the lock strands that define the chiral state of the origami template (Figure 1B $)^{24,25,26,31}$. For static assemblies, simpler block-shaped templates are often sufficient ${ }^{14,45,46,47}$.

The DNA origami-based approach to the fabrication of plasmonic nanostructure inherits limitations of the DNA origami technique ${ }^{48}$. The size of the origami templates is typically limited by the size of the scaffold strand. The stability of DNA structures is reduced under law-salt conditions. The cost of synthetic staple strands remains rather high. However, recent developments in the field of structural DNA nanotechnology are expected to overcome these limitations ${ }^{49,50,51,52,53,54,55}$.

Compared to other molecular-based approaches for generating chiral assemblies of AuNRs ${ }^{34,35,36,37}$, DNA origami provides a high level of spatial precision and programmability. 
For achieving reliable and reproducible optical responses of chiral assemblies, we strongly recommend adapting the protocols for AuNR synthesis $^{40}$, since the quality and optical properties of commercial products may vary between batches. Additional annealing (step 6.2) is often crucial for ensuring the correct attachment of AuNRs to DNA origami templates (Figure 6).

Finally, the protocol described here is not limited to chiral assemblies. DNA origami provides a very flexible platform for the fabrication of complex plasmonic nanostructures ${ }^{9,10}$.

\section{Disclosures}

The authors have nothing to disclose.

\section{Acknowledgments}

The authors thank S. Voutilainen for her assistance with CD spectrometer. The authors acknowledge the provision of facilities and technical support by Aalto University at OtaNano - Nanomicroscopy Center (Aalto-NMC). This work was supported by the Academy of Finland (grant 308992) and the European Union's Horizon 2020 research and innovation programme under the Marie Skłodowska-Curie grant agreement No. 71364.

\section{References}

1. Rothemund, P. W. K. Folding DNA to create nanoscale shapes and patterns. Nature. 440, 297-302 (2006).

2. Wang, P., Meyer, T. A., Pan, V., Dutta, P. K., Ke, Y. The Beauty and Utility of DNA Origami. Chem. 2, $359-382$ (2017).

3. Hong, F., Zhang, F., Liu, Y., Yan, H. DNA Origami: Scaffolds for Creating Higher Order Structures. Chemical Reviews. 117, 12584-12640 (2017).

4. Roller, E.-M., Argyropoulos, C., Högele, A., Liedl, T., Pilo-Pais, M. Plasmon-Exciton Coupling Using DNA Templates. Nano Letters. 16, 5962-5966 (2016).

5. Acuna, G. P. et al. Fluorescence Enhancement at Docking Sites of DNA-Directed Self-Assembled Nanoantennas. Science. 338, 506-510 (2012).

6. Roller, E.-M. et al. DNA-Assembled Nanoparticle Rings Exhibit Electric and Magnetic Resonances at Visible Frequencies. Nano Letters. 15 1368-1373 (2015).

7. Liu, Q., Song, C., Wang, Z.-G., Li, N., Ding, B. Precise organization of metal nanoparticles on DNA origami template. Methods. 67, 205-214 (2014).

8. Roller, E.-M. et al. Hotspot-mediated non-dissipative and ultrafast plasmon passage. Nature Physics. 13, 761-765 (2017).

9. Liu, N., Liedl, T. DNA-Assembled Advanced Plasmonic Architectures. Chemical Reviews. 118, 3032-3053 (2018).

10. Kuzyk, A., Jungmann, R., Acuna, G. P., Liu, N. DNA Origami Route for Nanophotonics. ACS Photonics. 5, 1151-1163 (2018).

11. Kuzyk, A. et al. DNA-based self-assembly of chiral plasmonic nanostructures with tailored optical response. Nature. 483, 311-314 (2012).

12. Shen, X. et al. Three-Dimensional Plasmonic Chiral Tetramers Assembled by DNA Origami. Nano Letters. 13, 2128-2133 (2013).

13. Liu, H., Shen, X., Wang, Z.-G., Kuzyk, A., Ding, B. Helical nanostructures based on DNA self-assembly. Nanoscale. 6, $9331-9338$ (2014).

14. Shen, X. et al. 3D plasmonic chiral colloids. Nanoscale. 6, 2077-2081 (2014).

15. Urban, M. J. et al. Plasmonic Toroidal Metamolecules Assembled by DNA Origami. Journal of the American Chemical Society. 138, 5495-5498 (2016).

16. Hentschel, M., Schäferling, M., Duan, X., Giessen, H., Liu, N. Chiral plasmonics. Science Advances. 3, e1602735 (2017).

17. Cecconello, A., Besteiro, L. V., Govorov, A. O., Willner, I. Chiroplasmonic DNA-based nanostructures. Nature Reviews Materials. 2, 17039 (2017).

18. Lan, X., Wang, Q. Self-Assembly of Chiral Plasmonic Nanostructures. Advanced Materials. 28, 10499-10507 (2016).

19. Shen, C. et al. Spiral Patterning of Au Nanoparticles on Au Nanorod Surface to Form Chiral AuNR@AuNP Helical Superstructures Templated by DNA Origami. Advanced Materials. 29, 1606533 (2017).

20. Zhou, C., Duan, X., Liu, N. A plasmonic nanorod that walks on DNA origami. Nature Communications. 6, 8102 (2015).

21. Douglas, S. M. et al. Rapid prototyping of 3D DNA-origami shapes with caDNAno. Nucleic Acids Research. 37, 5001-5006 (2009).

22. Kim, D.-N., Kilchherr, F., Dietz, H., Bathe, M. Quantitative prediction of 3D solution shape and flexibility of nucleic acid nanostructures. Nucleic Acids Research. 40, 2862-2868 (2012).

23. Maffeo, C., Yoo, J., Aksimentiev, A. De novo reconstruction of DNA origami structures through atomistic molecular dynamics simulation. Nucleic Acids Research. 44, 3013-3019 (2016).

24. Kuzyk, A. et al. Reconfigurable 3D plasmonic metamolecules. Nature Materials. 13, 862-866 (2014).

25. Kuzyk, A. et al. A light-driven three-dimensional plasmonic nanosystem that translates molecular motion into reversible chiroptical function. Nature Communications. 7, 10591 (2016).

26. Kuzyk, A., Urban, M. J., Idili, A., Ricci, F., Liu, N. Selective control of reconfigurable chiral plasmonic metamolecules. Science Advances. 3, e1602803 (2017).

27. Gerling, T., Wagenbauer, K. F., Neuner, A. M., Dietz, H. Dynamic DNA devices and assemblies formed by shape-complementary, non-base pairing 3D components. Science. 347, 1446-1452 (2015).

28. Zhou, C., Duan, X., Liu, N. DNA-Nanotechnology-Enabled Chiral Plasmonics: From Static to Dynamic. Accounts of Chemical Research. 50, 2906-2914 (2017).

29. Ijäs, H. et al. Dynamic DNA Origami Devices: from Strand-Displacement Reactions to External-Stimuli Responsive Systems. International Journal of Molecular Sciences. 19, 2114 (2018).

30. Lan, X. et al. DNA-Guided Plasmonic Helix with Switchable Chirality. Journal of the American Chemical Society. 140, 11763-11770 (2018).

31. Funck, T., Nicoli, F., Kuzyk, A., Liedl, T. Sensing Picomolar Concentrations of RNA Using Switchable Plasmonic Chirality. Angewandte Chemie International Edition. 57, 13495-13498 (2018). 
32. Zhou, C., Xin, L., Duan, X., Urban, M. J., Liu, N. Dynamic Plasmonic System That Responds to Thermal and Aptamer-Target Regulations. Nano Letters. 18, 7395-7399 (2018).

33. Zhan, P. et al. Reconfigurable Three-Dimensional Gold Nanorod Plasmonic Nanostructures Organized on DNA Origami Tripod. ACS Nano. 11, 1172-1179 (2017).

34. Ma, W. et al. Attomolar DNA detection with chiral nanorod assemblies. Nature Communications. 4, 2689 (2013).

35. Ma, W. et al. Chiral plasmonics of self-assembled nanorod dimers. Scientific Reports. 3, 1934 (2013).

36. Guerrero-Martínez, A. et al. Intense Optical Activity from Three-Dimensional Chiral Ordering of Plasmonic Nanoantennas. Angewandte Chemie International Edition. 50, 5499-5503 (2011).

37. Kumar, J. et al. Detection of amyloid fibrils in Parkinson's disease using plasmonic chirality. Proceedings of the National Academy of Sciences. 115, 3225-3230 (2018).

38. Stahl, E., Martin, T. G., Praetorius, F., Dietz, H. Facile and Scalable Preparation of Pure and Dense DNA Origami Solutions. Angewandte Chemie International Edition. 53, 12735-12740 (2014).

39. Shaw, A., Benson, E., Högberg, B. Purification of Functionalized DNA Origami Nanostructures. ACS Nano. 9, 4968-4975 (2015).

40. Ye, X. et al. Improved Size-Tunable Synthesis of Monodisperse Gold Nanorods through the Use of Aromatic Additives. ACS Nano. 6 , 2804-2817 (2012).

41. Near, R. D., Hayden, S. C., Hunter, R. E., Thackston, D., El-Sayed, M. A. Rapid and Efficient Prediction of Optical Extinction Coefficients for Gold Nanospheres and Gold Nanorods. The Journal of Physical Chemistry C. 117, 23950-23955 (2013).

42. Shi, D., Song, C., Jiang, Q., Wang, Z.-G., Ding, B. A facile and efficient method to modify gold nanorods with thiolated DNA at a low pH value. Chemical Communications. 49, 2533-2535 (2013).

43. Gür, F. N., Schwarz, F. W., Ye, J., Diez, S., Schmidt, T. L. Toward Self-Assembled Plasmonic Devices: High-Yield Arrangement of Gold Nanoparticles on DNA Origami Templates. ACS Nano. 10, 5374-5382 (2016).

44. Castro, C. E. et al. A primer to scaffolded DNA origami. Nature Methods. 8, 221-229 (2011).

45. Lan, X. et al. Bifacial DNA Origami-Directed Discrete, Three-Dimensional, Anisotropic Plasmonic Nanoarchitectures with Tailored Optical Chirality. Journal of the American Chemical Society. 135, 11441-11444 (2013).

46. Lan, X. et al. Au Nanorod Helical Superstructures with Designed Chirality. Journal of the American Chemical Society. 137, 457-462 (2015).

47. Zhu, C., Wang, M., Dong, J., Zhou, C., Wang, Q. Modular Assembly of Plasmonic Nanoparticles Assisted by DNA Origami. Langmuir. (2018).

48. Pinheiro, A. V., Han, D., Shih, W. M., Yan, H. Challenges and opportunities for structural DNA nanotechnology. Nature Nanotechnology. 6, 763-772 (2011).

49. Ducani, C., Kaul, C., Moche, M., Shih, W. M., Högberg, B. Enzymatic production of 'monoclonal stoichiometric' single-stranded DNA oligonucleotides. Nature Methods. 10, 647-652 (2013).

50. Praetorius, F. et al. Biotechnological mass production of DNA origami. Nature. 552, 84-87 (2017).

51. Wagenbauer, K. F., Sigl, C., Dietz, H. Gigadalton-scale shape-programmable DNA assemblies. Nature. 552, 78-83 (2017).

52. Zhang, T. et al. 3D DNA Origami Crystals. Advanced Materials. 30, 1800273 (2018).

53. Ong, L. L. et al. Programmable self-assembly of three-dimensional nanostructures from 10,000 unique components. Nature. 552, $72-77$ (2017).

54. Ponnuswamy, N. et al. Oligolysine-based coating protects DNA nanostructures from low-salt denaturation and nuclease degradation. Nature Communications. 8, 15654 (2017).

55. Agarwal, N. P., Matthies, M., Gür, F. N., Osada, K., Schmidt, T. L. Block Copolymer Micellization as a Protection Strategy for DNA Origami. Angewandte Chemie International Edition. 56, 5460-5464 (2017). 